\title{
A Multi-Functional Mobile Information System for Hospital Assistance
}

\author{
Dario Cavada \\ Independent Information Technology and Services Professional \\ dario.cavada.lab@gmail.com \\ Manfred Mitterer \\ Hospital Franz Tappeiner Meran-Merano \\ manfred.mitterer@asbmeran-o.it \\ Francesco Ricci \\ Free University of Bozen-Bolzano \\ francesco.ricci@unibz.it \\ Omar Moling \\ Free University of Bozen-Bolzano \\ omoling@gmail.com \\ Floriano Zini \\ Free University of Bozen-Bolzano \\ floriano.zini@unibz.it
}

\begin{abstract}
Ospedale Amico (Friendly Hospital) is a mobile and personalized information system, aimed at improving the quality of the communication between medical staff and patients. The system provides the patients with up-to-date and context dependent day hospital activity guidance, and let them enter personal data and browse user-adapted descriptions of their disease. In this paper we describe the system functionality an discuss how we have addressed the limitations identified in the field study of an earlier prototype.
\end{abstract}

\section{Introduction}

Personalized health proposes the adaptation of medical treatments and services to the characteristics, needs, and preferences of individual patients [10]. Mobile devices such as smartphones or tablets can play an important role in achieving these objectives, as they expand and improve the information flow between patients and clinicians $[4,5,9]$.

In this paper we present Ospedale Amico (Friendly Hospital), a multi-functional personalized mobile information system that leverages standard smartphones or tablets for providing a range of information and communication services to both patients and clinicians. The system was developed in tight cooperation with the personnel of the oncology unit of Meran-Merano hospital (South Tyrol, Italy) and focuses on three important aspects of the interaction between hospital and patients. The first aspect is the execution of the patients' day hospital schedules. To support this the system informs the patients about the time and location of their activities, prompts information about possible delays, and helps indoor navigation in the hospital by using simplified maps. The second aspect is the collection of a complete set of data describing the patients' conditions. This is important for identifying more precise diagnoses, carefully selecting medicines, and identifying tailored treatments. The third aspect relates to the need of increasing patients' awareness of their diseases. This has several benefits, including an improved ability to properly report their health condition, and to better understand the doctor's prescriptions.

Compared to the state of the art of mobile-based health interventions [5], Ospedale Amico introduces two novel features: supporting the execution of the patient schedule in the hospital; and using machine learning techniques to adapt the quantity of information to the patient's capability to assimilate it. Ospedale Amico improves an earlier prototype [6] that was extensively evaluated in the field with patients and clinicians [6, 7]. We illustrate how the usability issues of this previous system have been addressed and the new functionality implemented.

\section{Ospedale Amico Functionality}

Ospedale Amico is a healthcare mobile system providing a single entry point to several support functions. In addition to the guiding services provided by the previously mentioned earlier prototype, it includes an in hospital navigation tool, an adaptive component informing the patients about their diseases, and many clinical questionnaires. In addition, control panels for the clinicians allow them to manage the patients' schedule, their questionnaires, their clinical information, and the statistical elaboration of the questionnaires' data. 


\subsection{Services for Patients}

Hospital Guide. This service supports the patients while they are undertaking their day hospital schedule: a sequence of activities taking place in various wards and rooms. The patients can access in real time their schedules browsing the activities already performed and checking the status of the next one, i.e., the waiting time before it starts or if the patient has being already called for the activity (Figure 1-a). The system can visualize a map of the hospital and support the navigation to the room where the activity is scheduled (Figure 1-b) [12].

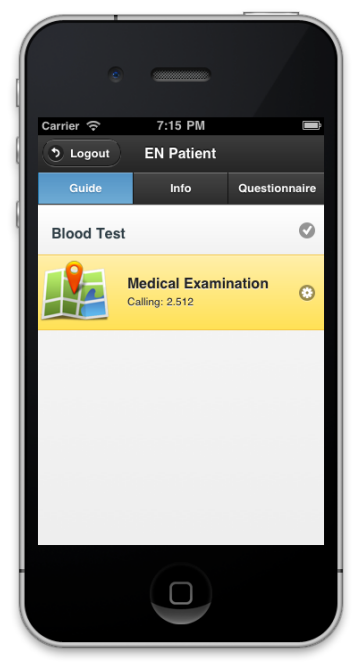

(a)

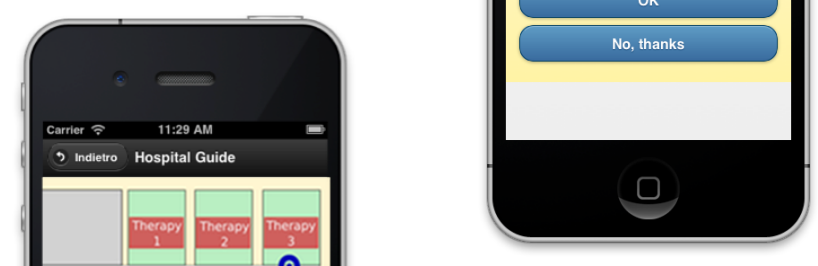

(a)

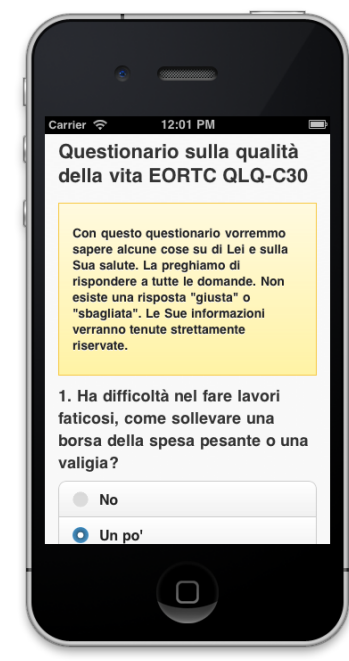

(b)
Figure 2. (a) Entertainment message; (b) Quality of life questionnaire filling.

Finally, reminder messages invite the patients to provide (via clinical questionnaires) information that is used by the doctor to better select or tune the therapy, and to read on their mobile devices documents that improve the knowledge of their diseases.

Questionnaire filling. Computerized questionnaires can effectively support the reliable collection of patients subjective clinical data [7, 8, 11]. Ospedale Amico supports the administration of two questionnaires. The first (EORTC QLQ-C30) is used to collect quality of life subjective assessment, with a special emphasis on socio-psycho-physical indicators [1]. The second monitors the side-effects of the prescribed medical treatments. In particular, we have focused on four common therapeutic protocols: FOLFOX (treatment of colorectal cancer), CISPLATIN (sarcomas, some carcinomas, lymphomas, and germ cell tumors), FEC (breast cancer), and $\mathrm{CHOP}$ (non-Hodgkin lymphoma). Patients can fill out their questionnaire on portable devices (Figure 2-b), then the data is processed and aggregated to be finally accessible to the clinicians (see Section 2.2).

Adapted clinical information. A system component called Personal Health Information System (PHIS) enables the patients to access documents containing information about their diseases, including the diagnosis, the evolution, the affected organs, and the possible treatments. PHIS aims at simplifying the patient's information acquisition process 


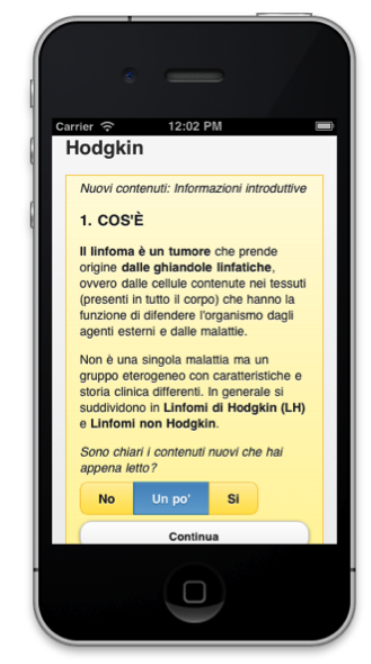

Figure 3. Presentation of a text block.

by presenting a document in a sequence of small text blocks (Figure 3). For this purpose, the available documents (31 in German and 23 in Italian) were logically decomposed in blocks of approximately equal size (100 words). All the blocks in a document were labeled with their main information type (e.g., diagnosis or disease evolution) and the logical dependencies among the blocks were identified. These dependencies are used to constrain the visualization: a block cannot be visualized until all the blocks it depends on have been shown and read. PHIS can use two visualization policies. The first one (baseline) visualizes with $90 \%$ probability the next block in the lexicographic sequence, while $10 \%$ of the times explores the space of alternative visualization sequences by randomly showing another dependent block. In this way some information is skipped and the amount of text shown to the patient is reduced. The system then registers if this has a positive or negative effect on content understanding by posing to the patient questions about the illustrated topics. In addition to the baseline policy, a second one, which is based on Reinforcement Learning [14], exploits the feedback provided by the patients to optimize the block presentation procedure.

\subsection{Services for clinicians}

One specific service is devoted to support the management of the patients' schedules. The nurses can assign to the patients a hospital schedule (among those predefined), change the patient's status, and enter delays information and reasons. These actions trigger appropriate messages sent to the patients. Another service enables the doctors to manage the questionnaires and clinical information to be sent to the patients. The doctors can assign clinical questionnaires to the patients, and browse their answers. Moreover, they can choose the clinical documents that must be provided to the patients. Finally, the system performs statistical elaboration of the questionnaires and produces easy to read charts. For example, Figure 4 shows the variation of the quality of life indicators in a time interval. Other charts illustrate the number of patients treated with the four considered oncological protocols in a time interval, or the variation over time of the side effect intensity experienced by a patient treated by these protocols.

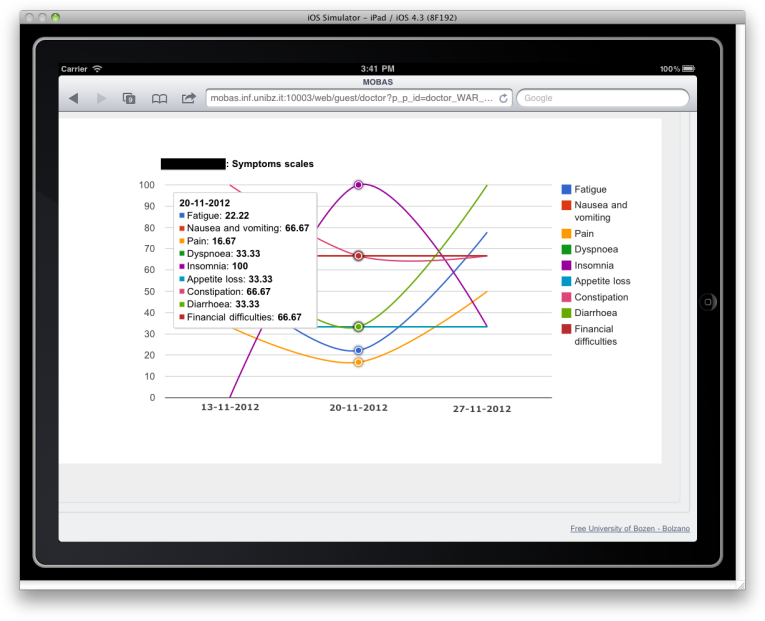

Figure 4. Variation of the quality of life indicators in a time interval.

\section{Discussion}

Ospedale Amico is substantially improving an earlier prototype [6] that we implemented as a J2ME application [2] for Nokia N97 touchscreen devices. This prototype was evaluated by the patients and clinicians of the same oncology unit of Meran-Merano hospital in two field studies $[6,7]$, which identified implementation and usability limitations that we have addressed in Ospedale Amico.

Since the earlier prototype was a J2ME native application, we had to provide to the patients special phones (Nokia N97) already equipped with the application. This forced the patients to learn how to use a new device and ultimately decreased the system usability [6]. For this reason we designed the new version as a mobile web application that is accessible via a standard web browser available on most smartphone and tablets. Hence, patients can now access Ospedale Amico using their own devices, and be immediately able to efficiently use the system. Moreover, the J2ME framework is now obsolete and that earlier prototype cannot run anymore on current popular mobile devices (iOS and 
Android). Moving to a web-based architecture enabled us to expand the usage base of our system. In addition, the adopted HTML5-based jQuery Mobile technologies [3] assure the portability of Ospedale Amico on all popular mobile device platforms.

During the tests of the first prototype we experienced many difficulties related to the accurate calibration of the RFID module used to localize the patients in the hospital. In addition, we discovered that the location of the patients did not provide a real additional benefit for customizing the system messages [6]. For these reasons we decided to discard the RFID module and base the message sending on the contextual factors mentioned in Section 2.1.

By analyzing the logs recorded in the first experimentation, we discovered that the nurses sometimes performed wrong schedule state changes and this triggered inappropriate messages. Therefore, in the new system we decided to more strictly constrain the nurses' actions to those requested by the patient's schedule. That was obtained with a new GUI for the nurses.

Finally, because of a limitation of the first prototype, about one third of the messages were actually read by the patients much later than the time they were received, i.e., when they were not useful anymore. Since we did not want to introduce auditive notifications in a sensitive environment as the hospital, we decided to adopt for the most important messages (call to a next activity) a multi-channel notification mechanism: they are now notified both on the patient's device and, in anonymous form, on a big wall display located in the waiting room.

It is worth noting that some features, such as the integration with the existing hospital information system, or the full patients' data safety, are not yet included in Ospedale Amico. This was agreed with the hospital staff in order to speed up the system development and focus the experimentation on the most innovative functions of the system.

\section{Conclusions}

In this paper we have described Ospedale Amico, an innovative day hospital mobile information system supporting the execution of the patients' hospital schedules by providing contextual guidance messages and a map functionality. The system also collects comprehensive data on the patients' health conditions by means of digital questionnaires and increases the patients' awareness of their diseases by introducing an adaptive information presentation component.

We are currently testing Ospedale Amico. We have conducted a pilot test involving few patients and clinicians and assessed that the system (hardware and software) works properly in the hospital. We are now running and extensive experimentation involving tens of patients and some clinicians. The collected data will be used to determine if
PHIS improves the patient knowledge of their disease and to assess the system usability and task completion rate, by comparing them with those of similar applications included in two benchmarks [13].

We plan to extend the system functionality to support the patients when they are at home or on the move. In particular, we want to explore how to suggest to the patients actions and behaviors that limit as much as possible the side effects of their therapies and lead to a better quality of life for them.

\section{References}

[1] EORTC group for research into Quality of Life. http: //groups.eortc.be/qol/index.htm, April 2013.

[2] Java platform, micro edition. http://en.wikipedia. org/wiki/Java_Platform,_Micro_Edition, April 2013.

[3] jquery mobile: Touch-optimized web framework for smartphones \& tablets. http://jquerymobile.com/, April 2013.

[4] J. E. Bardram. Pervasive healthcare as a scientific discipline. Methods of information in medicine, 47(3):178-185, 2008.

[5] P. Klasnja and W. Pratt. Healthcare in the pocket: Mapping the space of mobile-phone health interventions. Journal of biomedical informatics, 45(1):184-198, 2012.

[6] P. Lamber, B. Ludwig, F. Ricci, F. Zini, and M. Mitterer. Message-based patient guidance in day-hospital. In Mobile Data Management (MDM), 12th IEEE International Conference on. IEEE, 2011.

[7] P. Lamber, M. Mitterer, L. Napolitano, F. Ricci, and F. Zini. Surveying patients with smart devices. In Computer-Based Medical Systems (CBMS), 25th International Symposium on. IEEE, 2012.

[8] H. MacKenzie, A. Thavaneswaran, V. Chandran, and D. D. Gladman. Patient-reported outcome in psoriatic arthritis: a comparison of web-based versus paper-completed questionnaires. J Rheumatol, 38(12):2619-2624, Dec 2011.

[9] C. Orwat, A. Graefe, and T. Faulwasser. Towards pervasive computing in health care - A literature review. BMC Medical Informatics and Decision Making, 8:1-18, 2008.

[10] C. C. Y. Poon, M. D. Wang, P. Bonato, and D. A. Fenstermacher. Editorial: Special issue on health informatics and personalized medicine. Biomedical Engineering, IEEE Transactions on, 60(1):143-146, 2013.

[11] S. Ramachandran, J. Lundy, and S. Coons. Testing the measurement equivalence of paper and touch-screen versions of the eq-5d visual analog scale (eq vas). Quality of Life Research, 17:1117-1120, 2008.

[12] F. Ricci, G. Taraskeviciute, and F. Zini. Lightweight navigation in the hospital with portable devices. In ComputerBased Medical Systems (CBMS), 26th International Symposium on. IEEE, 2013.

[13] J. Sauro and J. R. Lewis. Quantifying the user experience: Practical statistics for user research. Morgan Kaufmann, 2012.

[14] R. S. Sutton and A. G. Barto. Reinforcement learning: An introduction. Cambridge Univ Press, 1998. 